\title{
The Project Method and investigation in school mathematics
}

\author{
Renáta Ujháziová, Ján Kopka, Dušan Šveda and Leonard Frobisher
}

\begin{abstract}
The Project Method (PM) is becoming more common in the teaching of mathematics. Most of the time, Project Method means solving open and relatively wide formulated problems for the application of particular mathematical topics and the solving of everyday life problems.

At present many experts in the theory of teaching mathematics advocate teaching activities as the characteristic for most mathematical work in the classroom. Thus, there is a question: whether it is possible or eventual desirable to use the PM for solving genuine mathematical problems. This paper deals with this question and discusses the connection between the PM and investigation of new mathematical knowledge for students. Our experience has shown that the PM in connection with investigations can be a useful and effective approach to teaching mathematics.
\end{abstract}

Key words and phrases: project method, investigation, problem solving, elementary theory of number, final digits, multiples of numbers, number systems.

ZDM Subject Classification: A05, C75, D05, D55, E55, F05, F35, F65, Q65.

\section{Introduction}

By Project Method (PM) we mean the solving of open and relatively wide formulated problems for the application of particular mathematical topic (for example: Angles around us and Functions in finance) and for solving real-life problems (for example: Savings or Travel by airplane). Many such problems are to be found in the references. In each of them the use of mathematics is often given just ex post. 
Our work at the Czech / Slovak research project is concerned with finding a connection between the PM and the investigation of new mathematical knowledge by students. That means, we wanted to realize projects, which have problem nature from the purpose point of view, which are based on the 'pure' mathematical problem and the character of the projects is divergent discovery work.

A necessary assumption for a successful use of the PM is thorough planning by the teacher of the solution stages as listed by Kilpatrick (Single 1992). This also holds for mathematical problems which students solve by investigation.

As part of the preparation a teacher should investigate the problem in order to be aware of possible outcomes. This enables the teacher to effectively interface at various stages of the of the students' investigation. We describe one project which we carried out during our research. The theme of this project is arithmetical, because arithmetics plays a very important role in Czech and Slovak mathematics education. This area of mathematics are well-known domain for students and also makes a very good possibilities for investigations, searching patterns, for developing problem posing and problem solving skills of students and mathematical processes, such as, predicting, conjecturing, hypothesizing, generalizing, proving, etc.

\section{Preparation of the project planning activity}

Name of the project: FINAL DIGITS OF MULTIPLES OF NUMBERS.

Age category: This project is designed for 15/16 years old students.

Topic: The project is related to ELEMENTARY THEORY OF NUMBERS and especially to the divisibility of natural numbers, greatest common divisor and least common multiple.

Pre-requisite knowledge: Knowledge of multiplication tables, how to determine the least common multiple and greatest common divisor.

Character of the project: Divergent discovery work.

Form of the output: Description of the output in written form.

Time framework: Two lessons ${ }^{1}$ (in the class).

\footnotetext{
${ }^{1}$ When we found out, how long it can be taken the necessary preliminary investigation with students and what is the real time-conditions of teachers in our schools, we decided to realize the common investigation just on two lessons. But students had whole one week for their independent investigation.
} 
Form of work: Students to work in pairs.

Intervention of the teacher: The teacher will direct student's works in minimum measure.

\section{Expectations:}

- To develop the ability of students to formulate and solve problems.

- To develop logical and creative thinking.

- To develop skills of students such as reasoning, arguing and proving.

- To give a chance to all students to feel happiness from doing mathematics.

- To encourage students to work in pairs.

\section{Educational aims:}

- Students find new properties of number.

- Students obtain some propaedeutic knowledge of the concept congruence.

Evaluation of students: Oral evaluation incorporating two possible criteria:

- Contents - number of formulated and checked problems - hypotheses.

- Form - presentation of the results according to a pre-determined model.

Evaluation of project: This takes the form of a student questionnaire.

\section{Plans for realization of the project:}

In Lesson 1 students will find the last digits of multiples 1 to 10 of the numbers from 1 to 10 . The principles of the evaluation of their work and the project will be explained to them. The lesson is completed with discussion of further problems which can arise from the initial investigation.

In Lesson 2 students will investigate properties of the set of final digits produced in Lesson 1 and will create and investigate new problems arising from the initial investigation.

\section{Mathematical content and structure of the investigation}

In this part we want to present the plan of investigation, which we prepared before realization of this project. Of course, this is just a hint and plan for us (for the teacher) and it is helpful for guiding the common investigation in some meaningful direction. But we do not present there all possible directions of investigation, which can be appeared during the investigation in the class. That's 
why this is just a guide for the teacher, which he can modify concerning the real investigation with students.

BASIC PROBLEM.

- Find the multiples 1 to 10 of numbers from 1 to 10 .

- Investigate the final digits of the multiples.

Expected outcome. The final digits of multiples are in Table 1.

Table 1

\begin{tabular}{|c|c|}
\hline Multiples & Final digits of multiples of numbers from 1 to 10 \\
\hline 1 & $1,2,3,4,5,6,7,8,9,0,1,2,3,4,5,6,7,8,9,0, \ldots$ \\
\hline 2 & $2,4,6,8,0,2,4,6,8,0, \ldots$ \\
\hline 3 & $3,6,9,2,5,8,1,4,7,0,3,6,9,2,5,8,1,4,7,0, \ldots$ \\
\hline 4 & $4,8,2,6,0,4,8,2,6,0, \ldots$ \\
\hline 5 & $5,0,5,0,5,0,5,0,5,0, \ldots$ \\
\hline 6 & $6,2,8,4,0,6,2,8,4,0, \ldots$ \\
\hline 7 & $7,4,1,8,5,2,9,6,3,0,7,4,1,8,5,2,9,6,3,0, \ldots$ \\
\hline 8 & $8,6,4,2,0,8,6,4,2,0, \ldots$ \\
\hline 9 & $9,8,1,8,5,2,9,6,3,0,9,8,1,8,5,2,9,6,3,0, \ldots$ \\
\hline 10 & $0,0,0,0,0,0,0,0,0,0, \ldots$ \\
\hline
\end{tabular}

Comment. Naturally, at first, students may write the whole multiples of numbers from 1 to 10 and then write the final digits of the multiples listed in Table 1.

Problem 1. Students investigate the final digits from the table and try to find some patterns. For example, they may compare the digits of multiples of numbers 4 and 6,2 and $8, \ldots$

Possible COnClusion. The final digits of each pair of multiples of 2 and 8 , 3 and 7,4 and 6,1 and 9 are the same, but occur in the opposite order.

Comment. Before Problem 1, sometimes it may be suitable to ask some less able students to investigate the final digits of the multiples of just one number, for example 4 or 6 . 
PRoBlem 2. Students may count how many final digits appear in each repeat of the sequence of each multiple of numbers from 1 to 10 . Does it hold any pattern? During the investigation we can see that it is enough to determine the numbers of various digits until the first occurrence of zero (including zero). The sequence then repeats itself. They may record this information in a table. See Table 2.

Table 2

\begin{tabular}{|c|c|c|c|c|c|c|c|c|c|c|}
\hline Multiple & 1 & 2 & 3 & 4 & 5 & 6 & 7 & 8 & 9 & 10 \\
\hline $\begin{array}{c}\text { Number of digits in each } \\
\text { repeat of sequence }\end{array}$ & 10 & 5 & 10 & 5 & 2 & 5 & 10 & 5 & 10 & 1 \\
\hline
\end{tabular}

Conclusion. Students can be encouraged to show each sequence of final digits diagrammatically. This could be by means of the so-called dial addition. We draw 10 hours in a circle dial, but instead of number 10 we write down just 0 . Figure 1 shows the circle dials for the multiples of 2 and 3.

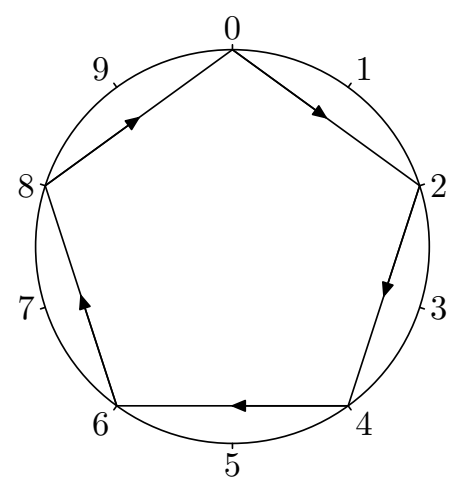

Sequence of final digits of multiples of 2

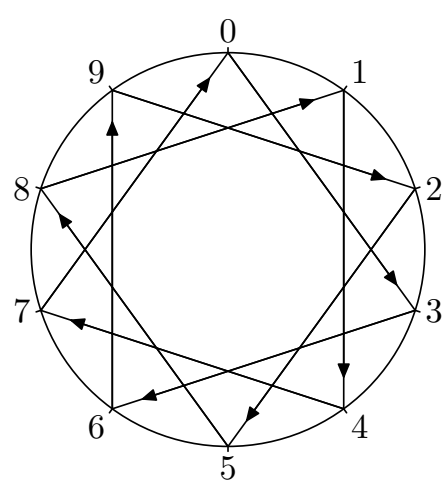

Sequence of final digits of multiples of 3

Figure 1

Question. How would you add numbers by means of this dial? 
We can add numbers in this way: For example we count the sum $8+8$ : at first we determine "the normal sum", which is 16 , and then we count 16 segments from the zero clockwise. We move to number 6 .

We can write: $8+8=6$. Likewise $(8+8)+8=6+8=4$, what is equivalent with notation $(3 \cdot 8)$.

Problem 3. Students may be asked to repeatedly add 8 to 8 , and to record and investigate the results.

RESULT. When we repeatedly add 8 to 8 , we obtain the final digits of the multiples of 8 . It will be analogical for other numbers from 1 to 10 , too. The mathematical name for dial addition is addition by modulo 10 .

Comment. This diagrammatic representation can help students determine the final digits of numbers in other number system.

Problem 4. Students may investigate patterns and relationships in Table 2.

RESULT. The multiples are collected into four sets according to the numbers of digits in each repeat of the sequence. $(1,3,7,9)$ have 10 digits in each repeat; $(2,4,6,8)$ have 5 digits; (5) has 2 digits and (10) has 1 digit.

Comment. We recommend that students investigate what is special about each set of numbers before they move on to Problem 5.

Problem 5. Students may investigate relationships between the numbers in each of the sets $(1,3,7,9),(2,4,6,8),(5)$ and (10) and the number 10 . They may be encouraged to try to formulate a general result as a hypothesis.

Comment. We recommend suggesting to students the direction of finding some patterns in this task.

Possible Result of inVEstigation.

The numbers 1, 3, 7, 9 have $\mathbf{1 0}$ final digits in each repeat and are also relatively prime with number 10 ; that means, for example, that $\operatorname{GCD}(3,10)=\mathbf{1}$.

The numbers 2, 4, 6, 8 have $\mathbf{5}$ final digits and are also are commensurable with number 10; that means, for example, that $\operatorname{GCD}(6,10)=\mathbf{2}$.

Similarly, 5 has $\mathbf{2}$ final digits and $\operatorname{GCD}(5,10)=\mathbf{5}$, and 10 has $\mathbf{1}$ final digit and $\operatorname{GCD}(10,10)=\mathbf{1 0}$. 
PROBLEM 6. Students may record the result of the investigation as a general statement, such as: For each number $x$ from 1 to 10 the number of final digits of multiples of $x$ multiplied by $\operatorname{GCD}(x, 10)$ is equal to 10 .

After Problem 6 students are asked to propose other problems, which lend themselves to further investigations.

POSSIBLE PROBLEMS FOR INVESTIGATION.

- Final digits of multiples of numbers $11,12,13, \ldots$

- Final digits of multiples of numbers in the number base 9 system.

- Final digits of multiples of numbers in the number base 12 system.

- Final digits of multiples of numbers from an arbitrary number base system.

4. Investigating the final digits in the multiples of numbers from 1 to 9 in the base 9 system

Table 3 shows the final digits of multiples in base 9 .

Table 3

\begin{tabular}{|c|c|}
\hline Multiples & Final digits of multiples of numbers from 1 to 9 in base 9 \\
\hline 1 & $1,2,3,4,5,6,7,8,0,1,2,3,4,5,6,7,8,0, \ldots$ \\
\hline 2 & $2,4,6,8,1,3,5,7,0,2,4,6,8,1,3,5,7,0, \ldots$ \\
\hline 3 & $3,6,0,3,6,0, \ldots$ \\
\hline 4 & $4,8,3,7,2,6,1,5,0,4,8,3,7,2,6,1,5,0, \ldots$ \\
\hline 5 & $5,1,6,2,7,3,8,4,0,5,1,6,2,7,3,8,4,0, \ldots$ \\
\hline 6 & $6,3,0,6,3,0, \ldots$ \\
\hline 7 & $7,5,3,1,8,6,4,2,0,7,5,3,1,8,6,4,2,0, \ldots$ \\
\hline 8 & $8,7,6,5,4,3,2,1,0,8,7,6,5,4,3,2,1,0, \ldots$ \\
\hline 9 & $0,0,0,0,0,0, \ldots$ \\
\hline
\end{tabular}

The number of digits in each repeat of the sequence of final digits is listed in Table 4.

The multiples are collected into three sets according to the numbers of digits in each repeat of the sequence. $(1,2,4,5,7,8)$ have 9 digits in each repeat; $(3,6)$ have $\mathbf{2}$ digits; and (9) has $\mathbf{1}$ digit. 


\section{Table 4}

\begin{tabular}{|c|c|c|c|c|c|c|c|c|c|}
\hline Multiples of number $x$ & 1 & 2 & 3 & 4 & 5 & 6 & 7 & 8 & 9 \\
\hline $\begin{array}{c}\text { Number of digits in } \\
\text { each repeat of sequence }\end{array}$ & 9 & 9 & 3 & 9 & 9 & 3 & 9 & 9 & 1 \\
\hline
\end{tabular}

The numbers $1,2,4,5,7,8$ have 9 digits and relatively prime with 9 ; that means, for example, $\operatorname{GCD}(4,9)=1$.

The numbers 3, 6 have $\mathbf{3}$ digits and are commensurable with 9; that means, for example, $\operatorname{GCD}(6,9)=3$.

For the number $9, \operatorname{GCD}(9,9)=9$ and it has one final digit.

We can now formulate a general result from the observations listed above: For each number $x$, from 1 to 9, in base 9, the number of final digits of multiples of $x$ multiplied by $\operatorname{GCD}(x, 9)$ is equal to 9 .

Similar results hold for multiples in an arbitrary base $z$, where $z$ is a natural number greater than 1. A consideration of each of the general statements for the different number bases results in a general statement of a higher order: For each number $x$, from 1 to $z$, in base $z$, the number of final digits of multiples of $x$ multiplied by $\operatorname{GCD}(x, z)$ is equal to $z$.

Comments. A reader, for whom the investigation of final digits of multiples of numbers in arbitrary number base systems is not enough, may wish to investigate the final digits of the even or odd numbers, triangle or square numbers, prime numbers, etc.

\section{Realization and evaluation of the project}

We performed this project over two lessons in two different high schools. In one school the students were specializing in mathematics; the two classes in the second school were non-mathematics students. The students were fifteen or sixteen years old in all classes. In the specialist mathematics class we worked on according to our plan. In the non-mathematics classes we were unable not conduct our project fully according our plan. There was insufficient time for independent investigation in pairs. These students worked much more slowly that the students in the mathematics class and it seemed that this kind of work was unusual for them. We gave them the directions and they were guided to results according to the contents of preparation and by means of common investigated patterns. 
All students had one week for the elaboration of their project, but they were no permitted to discuss any difficulties they encountered after the common investigation in the class.

During the independent investigation students mostly concentrated on investigating the final digits of square and cube of numbers from 1 to 10, of multiples of 1 to 10 in different number systems (mostly in the number system of 8 and 16) and of final digits of multiples of numbers 11 to 20 .

All students were able to begin to investigate the final digits of multiples in base 10 with some of them formulating the general hypotheses. Proving their own hypotheses and generalizations of the results proved to be the greatest difficulty for most students ${ }^{2}$. Some pairs were able to give reasons for the validity of their hypotheses, but others were unsuccessful in proving their hypotheses.

During lesson 1 students investigated some interesting patterns. Many pairs discovered the pattern: "If we count the final digits of multiples of the number for example 2 to the final digits of multiples of the number 8 , than we always obtain number 10." One pair formulated this pattern by writing: "The number 9 is a negation of the number." These students discovered intuitively the pattern of the opposite element, although they had no previous knowledge of its existence nor its characteristics.

Another interesting pattern discovered by some students relates to the multiples of 3 . The final digits of make the sequence of number $(3,6,9,2,5,8,1$, $4,7,0,3,6)$ in which the digits are lowered by 1 after each trinity. That means we can determine the second trinity of this sequence according to the first trinity lowering the value of each number by 1 . So the second trinity of numbers is 2 , 5 , 8. An analogical pattern holds for the multiples of 7 , but its final digits are increased by 1 . That means we can determine the second trinity of numbers from the first trinity of numbers, which are $7,4,1$, by adding 1 to each number of the first trinity. Thus, we obtain the trinity of numbers $8,5,2$.

During the common investigation some students discovered that if we take the final digits of, for example, the multiples of 8 , i.e. $(8,6,4,2,0,8,6,4,2,0)$, and we successively add the first number to the tenth, the second number to the ninth from this sequence, then we can see that final digits of these sums is always number 8. Analogical pattern holds for each final digit of multiples of number from 1 to 10 .

${ }^{2}$ These difficulties were confirmed not only from our observation, but from the questionnaire, too. 
Some students investigated unusual and creative problems during theirs independent investigation. Unfortunately, the most of students submitted us (in the written form) just a result of theirs investigations without description of the way, which leaded to create the hypothesis.

One pair's investigation resulted in the following:

Hypothesis. If we divide an arbitrary number from the ten number system with number 8 , then the rest of the division will be equal with final digits of this number in the number system of eight.

Comment. The hypothesis expresses indirectly, why we use just digits 0,1 , $\ldots, 7$ in the number system of base eight and it is very important from the point of view of students as it reinforces what they have learned about number system of base eight from computer lessons. They just know, that we use digits $0,1, \ldots$, 7 in this number system, but nobody had explained to them what the reason of this convention is. These students investigated this pattern by themselves. This is one of the most important aspects in the process of learning and experiencing new knowledge.

The same pair expressed the analogical hypothesis for the number system of four and generalized them this way: (We give them in the original formulation without corrections.)

GeneralizATION 1. If we divide the natural number $n$ in the number system of ten with number of number system to which we conduct, then the rest will be equal with the final digits of conducted number.

From the point of view of formulation the next generalization of this pair is also visible, although not correct.

Generalization 2. If we divide the number, which represent number system, from which the given number is, with GCP of given number and arbitrary natural number, we obtain the number of various final digits.

Another pair investigated additions and products of multiples of numbers from 1 to 10 and their final digits. This is what they wrote: The addition of multiples of two natural arbitrary numbers $a, b$ has so many final digits, how many is the smallest common multiple of final digits of multiples of numbers $a, b$. 
Hypothesis. The product of multiples of natural numbers $x, y$ has so many various final digits, how many final digits has the less number.

One problem, which was formulated and solved by one pair from the mathematical class, was remarkable, too. They formulated this problem: Find a formula, which helps us find final digits of arbitrary multiples of number from 1 to 10. A solution of this problem is contained in the next hypothesis.

Hypothesis. The final digit of multiple of arbitrary two factors from the small multiplication table is equal to difference between number 10 and the rest from the division $\frac{y \cdot(10-x)}{10}$, where $x, y$ are two arbitrary before declarable factors.

Comment. This hypothesis is in original formulation and its authors tried to prove it using words. But their proof is very tedious, complicated and incorrectly -arranged. Here we show just two examples of the hypothesis which may help to understand it.

Let $x=5, y=3$. We know that 5 multiplied by 3 is 15 . The final digit of this multiple is 5 . We can determine this final digit also from the above-mentioned formula this way:

First we determine the rest from the division $\frac{y \cdot(10-x)}{10}=\frac{3 \cdot(10-5)}{10}=\frac{15}{10}$. The rest from this division is 5 . Then we count the difference $10-5=5$. We can see, that the result of this difference is 5 , what is really the final digit of triples of number 5 .

When we choose for example $x=8$ and $y=9$, we can determine the final digit of multiple of these two numbers from the above-mentioned formula this way:

The rest from the division $\frac{y \cdot(10-x)}{10}=\frac{9 \cdot(10-8)}{10}=\frac{18}{10}$ is 8 and the difference $10-8$ is 2 . We know that 8 multiplied by 9 is 72 , and we can see that the final digit of this number is really 2 .

Another pair of students formulated a hypothesis, which is related to final digits of multiples of cubes of numbers 1 to 10 .

Hypothesis. If we divide the number 10 with the greatest common divisor of number $x$ and 10, where $x$ is number from 1 to 10, then we obtain the number of various final digits of multiples of that number.

As we mentioned above, the independent investigation of students were realized at home without possibilities to discuss any difficulties with us after the 
common investigation in the class. For this reason we could evaluate just the result of student's investigations, which they gave us one week later. We emphasized students, that the written form of theirs project has to contain:

(1) just problems, which are related to final digits of multiples of numbers, or final digits of numbers,

(2) exact formulations of problems (at least four of them for mark $1^{3}$ ), which was investigated and solved during the independent work,

(3) solving the formulated problems with creating the hypothesis and his proof ${ }^{4}$ or his verification at the particular examples,

(4) correct solutions (at least of two problems),

(5) (if it is possible) generalization of the results of investigation,

(6) transparent arranging the result of investigation,

(7) originality in problems posing.

We accomplished the evaluation of each projects for a consideration of these criteria, which was well known also for students. The students from the mathematical class were evaluated by words and marks, too, the students from another two classes just by words. In the mathematical class the students were noticed that their project will be awarding by marks and when they did not give over any work, they will aware marks $5^{5}$. In this class some students investigated very original problems, that's why we had to observe for this too. There for in such cases we evaluated the student's projects with the best mark, although these pairs of students solved less than four problems during theirs independent investigation.

At the evaluation of these projects we had to sense that the most of times the investigation of one original problem is more valuable than several investigations of analogical or similar problems. For this reason we think the originality in posing and investigating problems has to be all the same considerable criteria like the predetermined count of problems. That's why is not so easy to define the exact criteria for awarding marks. There is a big problem to make the qualitative determination of originality and creativity of investigation and express them by marks.

The different way of evaluation was reflected on the quality and the quantity of the results as well. While all students (expect for some exceptions) from the

${ }^{3}$ This count of problems referred just to students from mathematical class. For students from the another two classes were enough to investigate at least two problems.

${ }^{4}$ Students should prove theirs hypothesis at least by words.

${ }^{5}$ Mark 5 is the worst mark in our evaluation system. 
mathematical class gave us their results in shapely and written form, at another two classes just two projects accomplished our expectations. Our assumption that the evaluation by mark is important and mostly plumbless factor, which is affecting the achievement of students, was confirmed, although the questionnaire did not directly affirm even contradict our assumption.

The next factor, which in a great measure influenced the results of students, was the motivation of investigated topic. The answers (from the questionnaire) of the students from the non mathematics classes expressed their little interest in the topic of investigation. One student expressed his opinion this way: "The final digits - it does not have importance to vanish the time for them. I'd rather be thinking about the things, which I know about, that they will be useful in some field, for example in technology."

The students from the mathematical class showed more interest during the work on this project, probably to solve and investigate mathematical problems were not new for them. But here we have to remark that a few students from this class answered in the questionnaire, that they should be rather investigating in other fields that final digits. In spite of this topic was interesting for the majority of students. Just a few students answered in the questionnaire, that this project was no addition for them from the any point of view. One pair of students answered: "The addition of this project was not adequate to expended time, what I spent for it. I can imagine better ways how to develop my thinking." But another pair saw the addition of this project this way: "I know how to do the project in the future." Some students appreciate, that: "We could use own ideas during the investigation of topic."

\section{Conclusion}

The realized project accomplished our expectations in the mathematical class. The usual method of teaching in this class is often-investigative in character even before realization of this project. The connection between the investigation and the project method produced a large the quantity of original ideas and results which were created during the communication in pairs. The evaluation by marks was the strong motivation element.

In the non mathematical classes the realization of the project was only partly accomplished. This was the first experience that the students had with the project method and also their ability to investigate independently and to work in pairs to discover knowledge was at lower level. Their activity was reflected negatively by 
the fact that they would be evaluated just by words and not by marks (a fetish of marks as a motivation element in the students' learning, is regarded in generally as a negative phenomenon).

Our experience leads us to believe that project method in connection with investigations can be useful and effective, if:

- the project is prepared very well mainly from the mathematical contents point of view ${ }^{6}$,

- the topic is interesting and challenging for the students,

- the students have experience of the project method,

- the students have already encountered with some elements of investigations,

- there is a favorable climate in the class and enjoyment grows throughout the investigation.

\section{References}

[1] J. Kopka, The cluster of problems in school mathematics, University of J. E. Purkynianae, Ústí nad Labem, 1999 (in Czech).

[2] M. Kubínová and J. Novotná, Projects in mathematics education, in: Proceedings of CIEAEM 50, Neuchatel, 1998b.

[3] M. Kubínová, J. Novotná and G. H. Litter, Projects and mathematical puzzles - a tool for development of mathematical thinking, in: Proceedings of the First Conference of European Research in Mathematics Education (CERME 1), Forschungsinstitut für Didaktik der Mathematik, Osnabrück, 1999.

[4] M. Kubínová and M. Barešová, Teaching Mathematics as a suitable setting for developing communicative and co-operative faculties of pupils, in: Proceedings of CIEAEM 53, Verbania, 2001a.

[5] M. Ludwig, Projekte im Mathematikunterricht des Gymnasiums, Beiträge zum Mathematicunterricht, 1998 (in German).

[6] F. Single, American pragmatic pedagogy, Prague, SNP, 1992.

[7] M. Tichá and M. Kubínová, On the activating role of projects in the classroom, in: Proceedings of the First Conference of European Research in Mathematics Education (CERME 1), Osnabrück, 1999.

[8] J. Valenta and collective, Project method in the school and after school, in: Pohl'ady, Prague, IPOS ARTAMA, 1993 (in Czech).

${ }^{6}$ This is important for the teacher, because this enables him to be aware of possible outcomes and effectively interface at various stages of the of the students' investigation, also to judge the "quality of the student's projects", i.e. the quality of student's results achieved during the investigation. 


\author{
RENÁTA UJHÁZIOVÁ \\ INSTITUTE OF MATHEMATICS \\ DEPARTMENT OF ANALYSIS \\ P. J. ŠAFÁRIK UNIVERSITY \\ JESENNÁ 5 \\ 04154 KOŠICE \\ SLOVAKIA \\ E-mail: rujhaziova@yahoo.com
}

(Received May, 2005) 